\title{
QUELQUES MARQUEURS MORPHOLOGIQUES DES TEUCRIUM SECTION POLIUM (LAMIACEAE) DU MAROC. VALEURS DIAGNOSTIQUES À DIFFÉRENTS NIVEAUX D'INTÉGRATION
}

\author{
Jalal EL OUALIDI et Suzette PUECH
}

\begin{abstract}
RÉSUMÉ. Quelques marqueurs morphologiques des Teucrium section polium (Lamiaceae) du Maroc. Valeurs diagnostiques à différents niveaux d'intégration. La section polium du genre Teucrium (Lamiaceae) est constituée de groupes de taxons qui posent des problèmes au niveau détermination. Cette difficulté est la conséquence directe, pour un grand nombre de taxons, d'un manque de caractères stables à l'échelle de l'espèce. Cet article présente quelques marqueurs morphologiques, à différents niveaux d'intégration, dont la valeur taxinomique a été testée dans une étude biosystématique faite par nous et basée sur des critères multiples.

Parmi ces caractères diagnostiques nous citons le lobe latéro-postérieur et les poils tecteurs de la gorge de la corolle au niveau sous-section; les feuilles crénelées dès la base, les dents du calice mucronées au niveau groupe d'espèces ; la pilosité à la fois simple et bifide, la présence de racines adventives au niveau espèce polytypique; et bien d'autres caractères au niveau espèce non dimensionnelle (ou espèce très localisée) telles que la plagiotropie des axes secondaires et tertiaires de l'inflorescence, le squelette et l'architecture florale, la présence plus ou moins marquée des lobes latéro-postérieurs et la surface des akènes.
\end{abstract}

Mots clés. Teucrium section polium, biosystématique, marqueur morphologique, valeur taxinomique, niveau d'intégration, espèce polytypique, espèce non dimentionnelle .

ABSTRACT. Some morphological markers of Teucrium section polium (Lamiaceae) of Morocco. Diagnostical value at different integration. The section polium of the genus Teucrium (Lamiaceae) is composed of groups of taxa that set problems of determination. This difficulty is the direct consequence, for a great number of taxa, of a lack of stable characters at the level of the species. This paper presents some morphological markers at different integration levels, the taxonomic value of which was tested in a biosystematic study we carried out, based on multiple characters.

Among these diagnostic characters we quote the proximal lobes and the trichomes of the throat of the corolla at the level of the subsection; the leaves crenellated from the base, the shape of the calyx at the level of the group of species ; the simple and bifide trichomes at once, the presence of adventive roots at the level of polytypical species; and some other characters at the level of non-dimensional species (or reduced-areas species) such as the plagiotropism of secondary and tertiary axes of inflorescence, the flower architecture, the presence more or less marked of proximal lobes and the seed surface.

Key words. Teucrium section polium, morphological marker, taxonomic value, integration level, polytypical species, unidimensional species, biosystematic. 


\section{INTRODUCTION}

La systématique phénétique, science uniquement basée sur des critères morphologiques apparents, ne permet pas toujours d'approcher ou d'interpréter des phénomènes évolutifs, surtout quand il s'agit de complexes d'espèces comme c'est le cas pour la section polium du genre Teucrium.

Il est donc nécessaire de recourir à des études biosystématiques qui tiennent compte de la variabilité à l'intérieur de l'espèce et qui par conséquent conduisent à la délimitation des taxons très proches des espèces biologiques (Mayr, I963).

Par ailleurs, le systématicien est confronté à deux réalités difficilement conciliables : (1) les données fournies par l'étude biosystématique lui permettent de dresser une classification basée sur des critères hiérarchiques multiples. (2) Mais une étude systématique est censée également fournir une clé de détermination des différents taxons : le systématicien élabore ici, une classification basée sur des critères purement morphologiques qui doivent la rendre directement accessible à l'utilisateur.

Cependant, il est souvent difficile de trouver des caractères, à la fois morphologiques et diagnostiques, surtout quand on sait qu'une différence dans le phénotype ne traduit pas forcément un isolement sexuel et que l'interaction génotype-environnement cache bien souvent des caractères plastiques à l'échelle de l'espèce mais stable au niveau de la population. Ainsi l'étude des cultures contrôlées donne une bonne estimation de la plasticité phénotypique ou norme de réaction du génotype (Bradschaw, I965 ; Stearns, I989), elle sépare donc les caractères ayant une valeur adaptative des caractères stables.

La liste de marqueurs que nous allons présenter, concernant la section polium du genre Teucrium du Maroc, est basée sur une étude biosystématique (El Oualidi, 1991), comprenant l'analyse de la pilosité et de la surface des akènes en microscopie électronique à balayage, les études chimiotaxinomique, caryologique et cytométrique, l'étude de certains paramètres de croissance chez les plantules en phase exponentielle et en conditions contrôlées, les études de L'architecture florale et inflorescentielle, de la biologie de la reproduction et d'une approche de génétique écologique. Nous n'avons présenté que certains de ces marqueurs, concernant seulement l'aspect macro morphologique accessible de manière directe et facile pour la détermination des taxons. Nous avons retenu dans cet article uniquement les résultas bruts avec un minimum de commentaires.

Bien que ces marqueurs concernent plus particulièrement les Teucrium marocains, ils peuvent très bien servir pour regrouper dans les mêmes complexes d'autres taxons du bassin méditerranée occidental, notamment ceux de la péninsule ibérique qui montrent une plus grande diversité et posent souvent des problèmes de détermination. Dans un deuxième temps, l'étude que nous avons effectué sur les Teucrium du Maroc s'insère dans une perspective biogéographique et intègre les travaux réalisés sur ce même groupe (Puech, 1976, 1980, 1990 ; Navarro, 1988).

\section{RESULTÉS}

\section{Historique et présentation de la section polium}

Dans le genre Teucrium, Bentham (I932I936) a fondé le découpage en sections sur la forme de l'inflorescence et celle du calice. Parmi les neuf sections distinguées, il caractérise la section polium par son inflorescence condensée en «capitules» terminaux, par son calice subrégulier et par sa corolle à lobe antérieur oblong, décliné et à lobes latéraux petits. Dans la section polium, il 


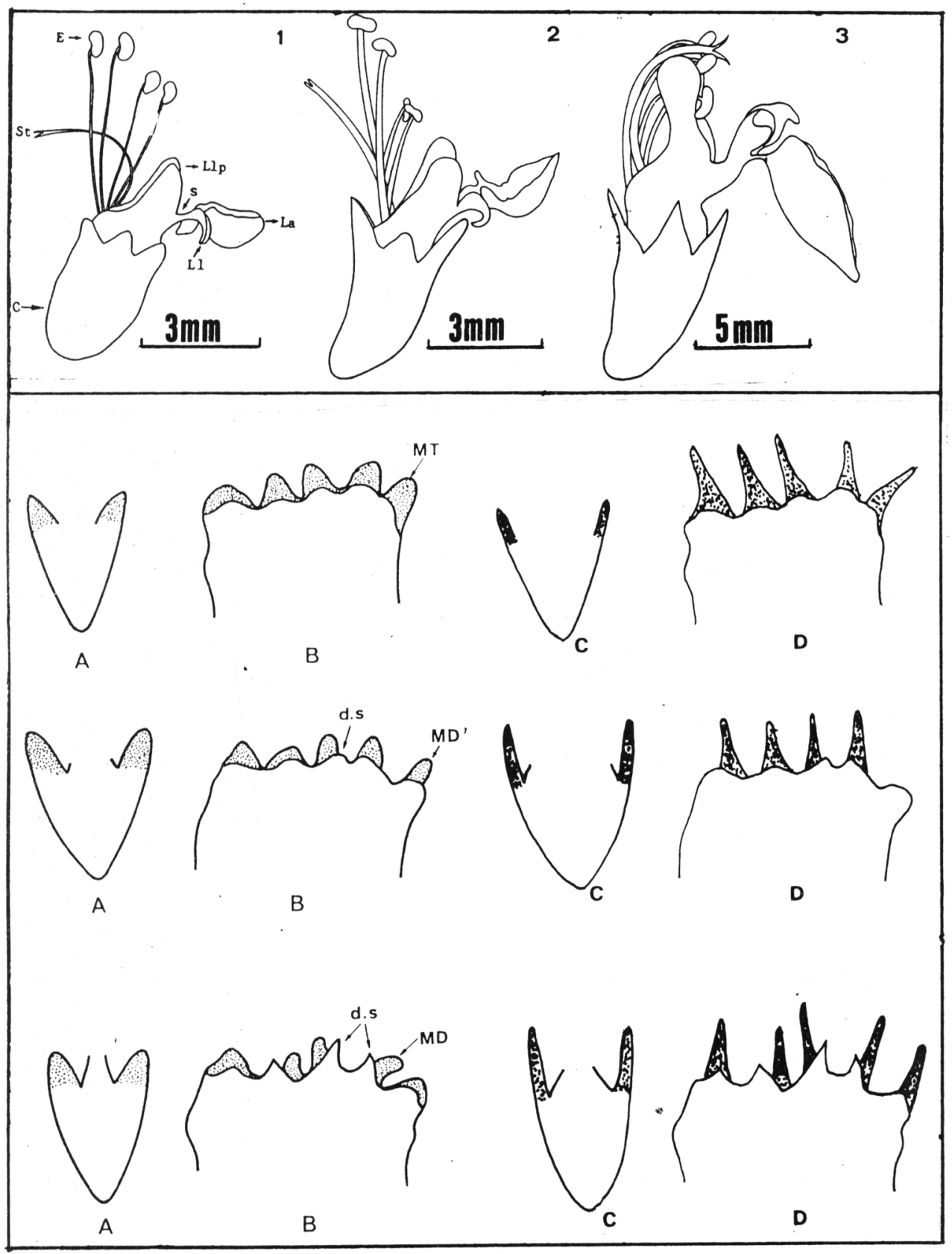

Figure 1. Architectures florales du Type T. polium $(1,2)$, du type $T$. rotundifolium (3) et schéma de calices du type T. puechiae et T. mideltense (A,B) et du type T. mairei (C.D): 1: T. mairei; 2: T. puechiae; 3: T. rotundifolium; c: calice; St: style; E: étamine; Llp; lobes latero-postérieurs; Ll: Lobes látéraux; La: Lobe antérieur; S: intersection entre llp et $\mathrm{Ll}$ sur la projection. A,C: coupe longitudinale du calice; B,D: calice ouvert; MT: mucron terminal; MD': mucron subterminal; MD: mucron dorsal; ds: dents des sépales. 
distingue deux groupes, l'un à feuilles crénelées (Teucrium polium, T. rotundifolium) et l'autre à feuilles entières ( $T$. thymifolium, $T$. montanum, $T$. pumilum).

Briquet (I891) n'utilise que la forme de l'inflorescence, il différencie la section polium des autres sections par les verticillastres (ensemble de cymes portées par les axes florifères secondaires formant ainsi un faux verticille) condensés au sommet des axes en «capitules», l'inflorescence n'étant jamais dorsiventrale (inflorescence dorsiventrale = ensemble de fleurs se regroupant en avant, laissant à nu la face dorsale).

Cohen (I956) a combiné les deux clés des sections et a séparé la section polium des cinq autres sections uniquement sur la base de l'inflorescence non dorsiventrale.

La section polium au Maroc est particulièrement riche, (dixneuf espèces) et constitue (selon Sauvage et Vindt, I955) deux groupes bien distincts : la sous-section Rotundifolia (dix espèces) à indument des tiges comprenant des poils simples et la sous-section polium à indument des tiges comprenant des poils tecteurs ramifiés. En plus de ces deux sections, Cohen (I956) a constitué la sous section Intermedia dont les espèces présentent un indument comprenant à la fois des poils tecteurs simples et ramifiés. Cette dernière comprend deux espèces que Sauvage et Vindt avaient mises dans la sous-section Rotundifolia.

Cependant, les critères retenus par ces auteurs négligent certains caractères floraux qui s'avèrent exclusifs pour quelques groupes d'espèces indépendamment de la forme de l'inflorescence et de la pilosité, comme par exemple la fleur et notamment la taille des lobes latéro-postérieurs qui suffit à distinguer la sous-section polium des autres taxons du genre Teucrium.

Ainsi, la clé publiée par Sauvage et Vindt (I955, I965), basée sur une étude purement morphologique, est incontestablement insuffisante et mérite un appui biosystématique, surtout dans un groupe aussi naturel et dispersé que la section polium. Néanmoins, cette clé rend bien compte de la grande variabilité à l'intérieur de la section, et permet donc dans certains cas de repérer les caractères variables au sein d'une espèce.

Les critères de base pris en compte pour trancher entre les espèces concernent le calice : l'intérieur glabre ou velu; dents courtes ou longues, aiguës ou obtuses ; présence ou absence de mucron, alors que beaucoup de caractères touchant au calice montrent un aspect polymorphe ou plastique. (El Oualidi, I987, I991).

La clé de Sauvage et Vindt, contrairement à celle de Puech (I976), n'intégre pas la pilosité du lobe postérieur, caractère relativement plus stable, par conséquent la clé montre ici moins de rigueur et donc moins de cohérence avec sa démarche générale.

Certaines espèces pourraient être distinguées dès le départ de la clé car elles présentent des caractères spécifiques et propres dans la section polium. C'est le cas par exemple des poils tecteurs chez $T$. mideltense (Batt.) Maire, et du lobe latéro-postérieur chez $T$. gypsophilum J. Emb. \& Maire.

Par ailleurs il existe à l'intérieur de la section polium des groupes ou complexes d'espèces au sein desquels les taxons sont très peu différenciés ; suite à une radiation adaptative récente, ces taxons témoignent d'une spéciation en cours de réalisation, caractérisée par une ambiguïté taxinomique, due à un chevauchement de caractères et par conséquent à un manque de marqueurs stables à l'échelle de l'espèce.

Parmi ces complexes nous citons:

1. Les Teucrium sous section polium à haut niveau de ploïdie et à mucron, présents dans le bassin méditerranéen occidental (Puech, I976, 1980, 1984 ; Navarro, I988 ; El Oualidi, I991). Il s'agit du complexe T. polium L - T. mairei Sennen (sensu lato) - T. puechiae Greuter \& Burdet (sensu lato). Au Maroc, seul 


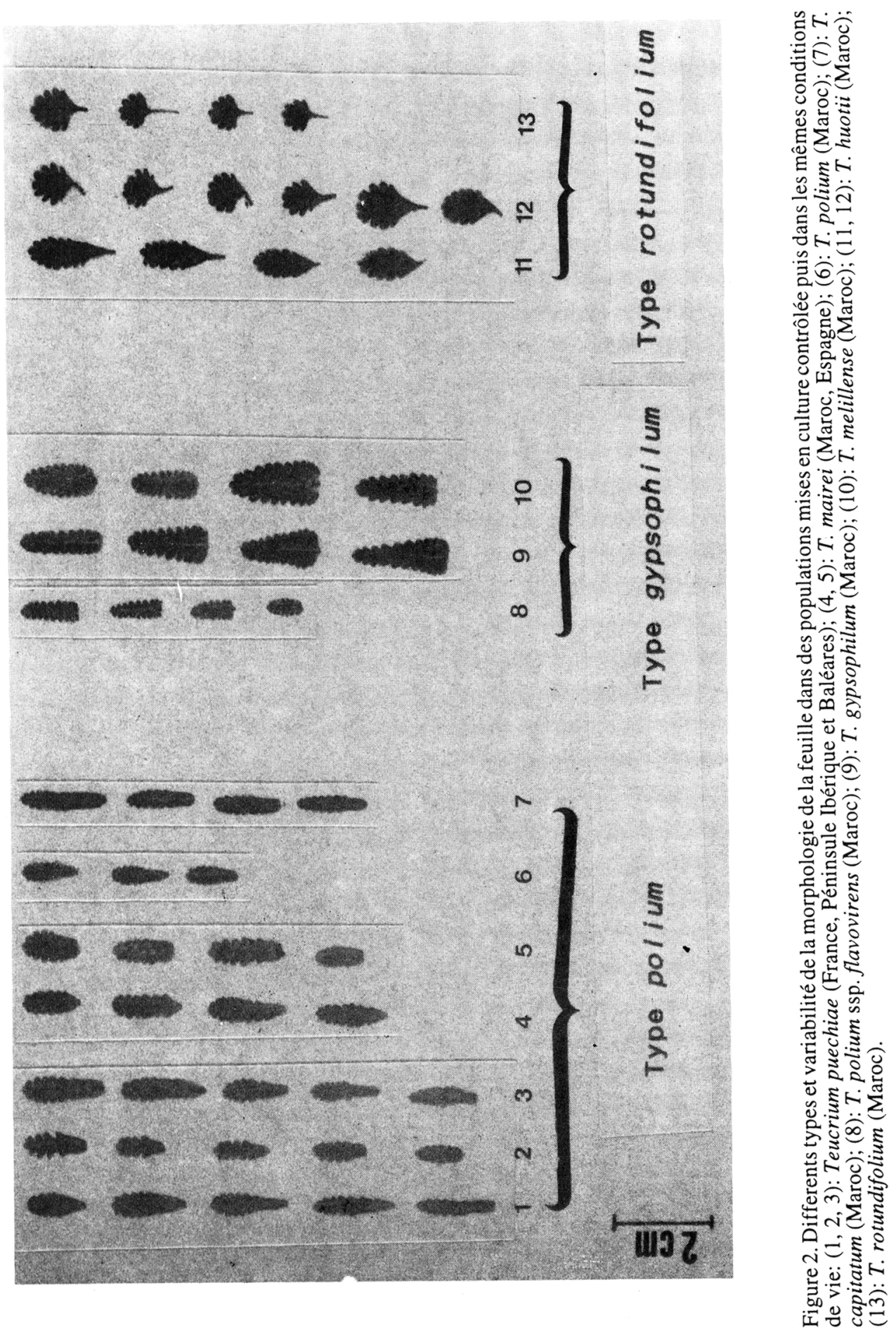


le complexe $T$. polium L.- T. mairei Sennen (sensu lato) existe, dans la moitié nord du Maroc (Rif et Moyen Atlas).

2. Le complexe T. mesanidum (Litard. \& Maire) Sauvage \& Vindt - T. cylindricum (Maire) Sauvage \& Vindt - T. polium L., où le passage d'une espèce à une autre présente beaucoup de formes intermédiaires. Ce complexe est bien réparti du nord au sud du Maroc. La forme typique du $T$. cylindricum occupe la partie méridionale de l'aire de répartition (Maroc saharien, selon les subdivisions géographiques adoptées par Fennane et al., 1986). Alors que le T. polium, occupe plutôt la partie nord de l'aire.

3. Le complexe $T$. melillense Maire \& Sennen - T. chlorostachyum Pau et Font-Quer, où il n'existe entre les taxons que très peu de différences morphologiques; ils sont très localisés mais ne présentent pas de barrières géographiques.

4. Le complexe polyploïde au sein du $T$. capitatum (L.) Arc., l'espèce la plus répandue et la plus polytypique du Maroc. Il existe à la fois des populations $(2 n=2 x)$ et des populations $(2 n=4 x)$ occupant des milieux bien particuliers.

5. Le complexe T. huotii J. Emb. et Maire, où il existe une variation géographique très importante de part et d'autre du massif rifain ; celle-ci témoigne d'une spéciation allopatrique récente.

6. Le complexe T. rotundifolium Schreb. - T. musimonum Humbert, qui occupe des biotopes diversifiés et présente une large répartition. C'est aussi, le groupe qui montre le plus d'affinité avec le complexe $T$. huotii à cause de son polytypisme. La forme typique du T. musimonum, est la plus montagnarde de toutes.

\section{Quelques marqueurs morphologiques.}

\section{Au niveau section.}

L'inflorescence est en faux capitules et n'est pas dorsiventrale (Bentham, 1932-1936).
2. Au niveau sous-section.

Les lobes latéro-postérieurs (LLP) sont très développés, égalant au moins (bien entendu chezles individus hermaphrodites) les anthères, chez la sous-section Rotundifolia les deux lobes étant convergents et jointifs, contrairement à la sous-section polium où ils sont courts, et permettent aux anthères de se dégager (fig. $1.1,1.2,1.3)$.

Feuilles rondes ou légèrement ovales, toujours nettement pétiolées chez la soussection Rotundifolia (fig. 2).

Les poils tecteurs des feuilles et des tiges sont tous simples chez la sous-section Rotundifolia.

Les poils tecteurs de la gorge de la corolle sont nettement plus gros et contrastent avec le reste de la pilosité, ils peuvent être à section plate ou ronde ; ces poils existent chez la soussection Rotundifolia (fig. 3a1, 3a2).

Le calice chez la sous-section Rotundifolia est à dents divergentes à maturité.

\section{Au niveau groupe d'espèces}

La feuille est crénelée dès la base, elle est \pm subamplexicaule, c'est le cas des $T$. gypsophilum, T. melillense, T. chlorostachyum et du $T$. polium ssp. flavovirens Sauvage et Vindt (fig. 2).

Les poils tecteurs de la gorge sont nettement plus gros et plats, c'est le cas des $T$. huotii des Bokkoyas et du Rif, du T. buxifolium et du $T$. musimonum.

L'existence d'un mucron au niveau des dents du calice. Le caractère mucron est très polymorphe et peut également présenter des différences qualitatives selon les taxons (fig. 1.A, 1.B, 1.C, 1.D), c'est le cas du complexe $T$. mairei au Maroc.

\section{Au niveau espèce polytypique}

La présence de racines adventives chez $T$. polium ssp. flavovirens est stable, l'abondance de ces racines est fonction du port, plus ou moins prostré. 


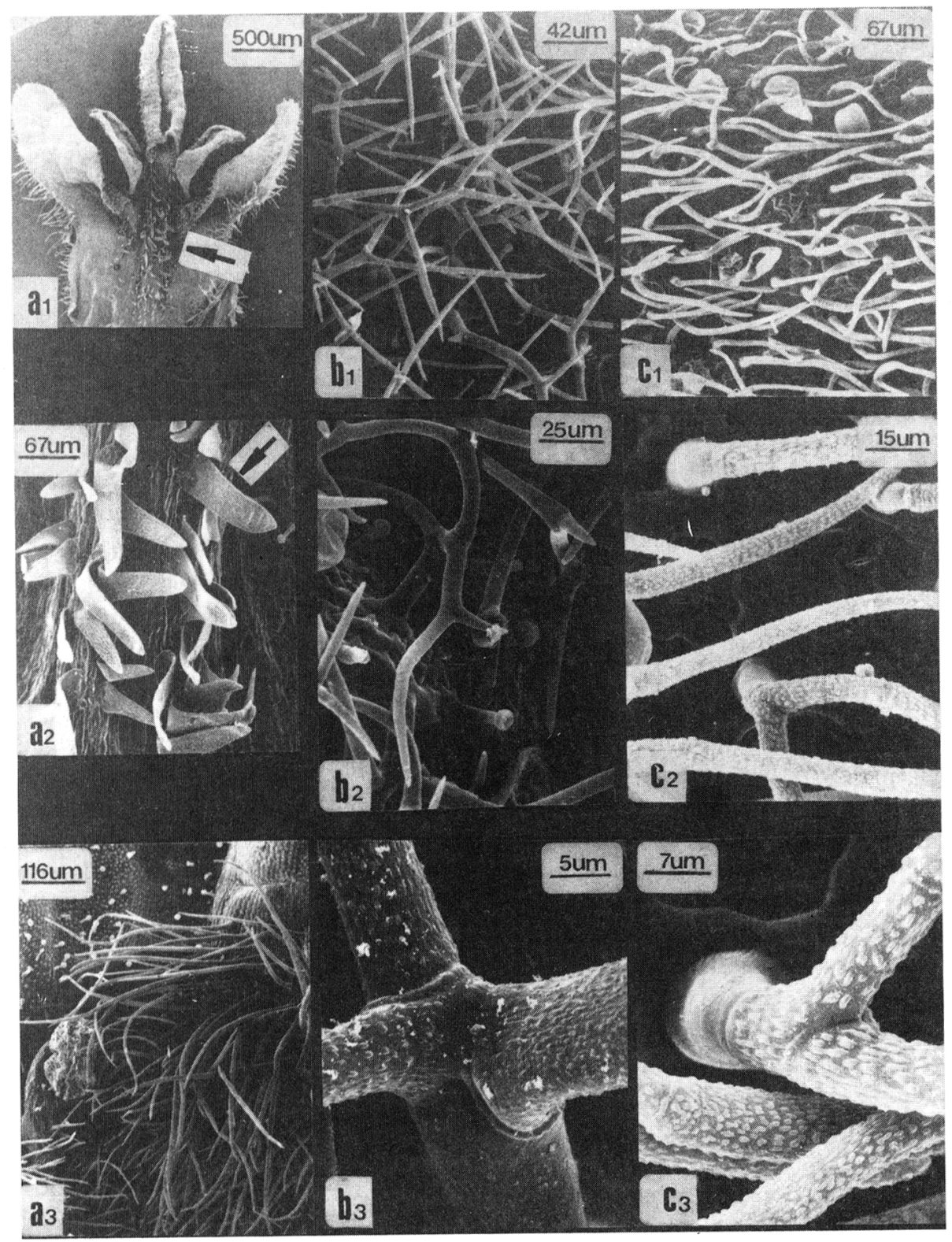

Figure 3. Détails de la pilosité en microscopie électronique à balayage: a1: gorge de la corolle de Teucrium huotii; a2: poils tecteurs simples et plats de la gorge de la corolle, typique de la sous sect. Rotundifolia; a3: poils tecteurs simples et ronds de Teucrium melillense, typique de la sous sect. polium. b1, b2, b3: pilosité de la face supérieure de la feuille du type $T$. polium (poils tecteurs longs et ramifiés à plusieurs niveaux); $\mathrm{c}_{1}, \mathrm{c}_{2}, \mathrm{c}_{3}$ : pilosité de la face supérieure de la feuille de T. mideltense (poils tecteurs simples ou bifides dés la base, toujours apprimés 
La pilosité courte, à la fois simple et bifide dès la base, et apprimée antrorses, caractérise $T$. mideltense sur toute son aire de distribution (fig. $3 \mathrm{c} 1,3 \mathrm{c} 2,3 \mathrm{c} 3$ ).

5. Au niveau espèce non dimentionnelle (ou espèce très localisée)

Il existe au Maroc des espèces de Teucrium à distribution très restreinte, réduite à une seule localité : c'est le cas de la majorité des endémiques. Ces espèces manifestent très peu de variabilité intraspécifique et offrent suffisamment de marqueurs morphologiques stables ; c'est le cas par exemple des lobes latéro-postérieurs très réduits et de la dominance apicale chez T. gypsophilum; de la plagiotropie des axes secondaires et tertiaires de l'inflorescence et de la surface plus lisse des akènes chez T. huotii.

\section{CONCLUSION}

L'étude des Teucrium du Maroc (El Oualidi, 1991) contribue à aborder de manière globale et synthétique les problématiques concernant les taxons à large répartition, présents au Maroc ou ailleurs dans le bassin méditerranéen occidental et à regrouper les espèces affines dans des complexes taxinomiques plus facilement lisibles et interprétables.

En effet dans le sud est de la Péninsule Ibérique, véritable foyer de la radiation, l'abondante variation phénotypique à l'intérieur de certaines espèces (Puech, 1976, 1984 ; Navarro, 1988), témoigne de la nécessité à réviser le statut des espèces en prenant en considération les taxons nord africains et à harmoniser les méthodes d'approche.

En revanche, la section polium au Maroc est constituée, (1) d'une part de taxons ayant été piégés dans des milieux très spécialisés, de ce fait leur isolement a limité le flux génique (Slatkin, 1987) et favorisé une spéciation rapide, selon les théories classiques de l'adaptation (Maynard Smith, 1988 ; Stace, 1988) ; c'est le cas des Teucrium endémiques à distribution restreinte et qui de ce fait ne présentent pas beaucoup de variabilité et ne posent donc pas de problèmes de détermination. (2) D'autre part, cette même section comprend des taxons très répandus et occupant des milieux différents ; ils représentent des unités à la fois infra- et supra-spécifiques, et forment ainsi des complexes ou groupes d'espèces qui ne peuvent être étudiés qu'en attachant de l'importance à tous les taxons d'un même groupe, aussi bien au Maroc que sur l'aire entière de sa répartition.

Il est vraisemblable que la différenciation des taxons à l'intérieur de la section polium est plus avancée au Maroc qu'en Péninsule Ibérique (peu de complexes d'espèces), ceci est vraisemblablement dû aux multitudes de niches écologiques qu'offre le territoire marocain (très large ditribution), loin de l'origine de la radiation adaptative, d'où la nécessité d'inscrire cette étude dans une perspective purement évolutive du groupe et qui aura pour but d'harmoniser les approches, les nomenclatures des taxons existant en région méditerranéenne et bien entendu la réduction éventuelle du nombre d'espèces.

\section{BIBLIOGRAPHIE}

BENTHAM, G. -1832/1836- Labiatarum genera et species or A description of the genera and species of plants of the order Labiatae. Ridgway \& sons, London, 783p.

BRADSHAW, A.D. -1965- Evolutionary significance of phenotypic plasticity in plants. Adv. Genet., 13: 115-155.

BRIQUET, J. -1891- Les Labiées des Alpes maritimes Georg., Genève, 587pp.

COHEN, E. -1956- Contribution à l'étude des Teucrium marocains de la section polium. Trav. Inst. Sci. Chérifien. Sér. Bot., 9, 88p.

EL OUALIDI, J. -1987-Approche de l'étude biosystématique des Teucrium de la section 
polium (Lamiaceae) du Maroc. Contribution à la «Flore pratique du Maroc». DEA Sciences de l'évolution et écologie, USTL, 91p.

EL OUALIDI, J. -I991-Biosystématique et taxinomie des Teucrium de la section polium (Lamiaceae) dans le bassin méditerranéen occidental. Différents aspects de la variation au Maroc, en France et en Espagne. Thèse Doct., USTL, Montpellier II, 22Op.

FENNANE, M., J. MATHEZ, A. OUYAHYA \& C. RAYNAUD -1986- Eléments pour la Flore pratique du Maroc, fasc. 1. M. FENNANE \& J. MATHEZ ed. Naturalia Monsp. sér. Bot., 50:552

MAYNARD SMITH, J. -1989- Evolutionary genetics. Oxford University Press, Cambridge, $325 \mathrm{p}$.

MAYR, E. -1963- Animal species and evolution. Harvard Univ. Press, Cambridge, Massachussets, 453p.

NAVARRO, T. -1988- Estudios biosistemáticos en el género Teucrium (Sección polium subsección polium) en la Península Ibérica (Lamiaceae). Tesis Doctoral Univ. de Granada, Fac. de Ciencias, 2: 173+171p.

PUECH, S. -1976- Recherches de biosystématique sur les Teucrium (Labiées) de la section polium du bassin méditerranéen occidental (Espagne et France). Thèse Doct. Sci., U.S.T.L., Montpellier, 138p.

PUECH, S. -1980- Les Teucrium de la sect. polium aux iles Baléares (Majorque). Bull. Soc. Bot. Fr., 127, Lett. Bot., (1): 41-52.

PUECH, S. -1984- Les Teucrium (Labiées) de la sect. polium du bassin méditerranéen occidental (France et péninsule ibérique). Naturalia Monsp., A5, 71p.

PUECH, S. -1990- Contribution à l'étude de biosystématique des Teucrium de la section polium (Labiatae) de Tunisie III. Bull. Soc. Bot., 137, Lett. Bot., (1) : 63-76.

SAUVAGE, Ch. \& J. VINDT -1955- Synopsis du genre Teucrium Sect. polium au Maroc. Bull. Soc. Sc. Nat. Phy. du Maroc, 35: 283-293.

SAUVAGE, Ch. \& J. VINDT -1965- Synopsis du genre Teucrium Sect. polium au Maroc (suite et fin). Naturalia Monsp., 16: 195-200.

SLATKIN, M.K. -1987- Gene flow and the geographic structure of natural populations. Science, 236: 787-792.
STACE, C.A. -1988- Plant taxonomy and biosystematics. Edward Arnold 2nd.ed 264p.

STEARNS, S.C. -1989- The evolutionary significance of phenotypic plasticity. Phenotypic sources of variation among organisms can be described by developmental switches and reaction norms. Bio Science, 39(7): 436-445.

Aceptado para su publicación en Agosto de 1993

Adresse des auterurs. J. El Oualidi: Institut Scientifique, Univ. Mohammed V, Rabat, Agdal, BP 703, Maroc. S Puech: Institut de Botanique, Univ. SC. Tech., Montpellier, France. 